
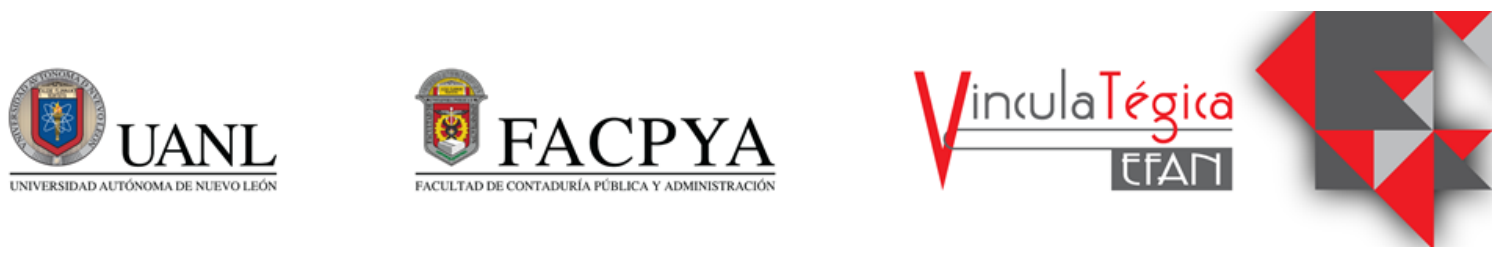

\title{
Voz inteligente para la capacitación del profesionista en el paradigma 4.0
}

\author{
Stephanny Balderas-Ramírez ${ }^{1}$; Nora Odalyz Barragán-Hernández ${ }^{2}$ y Lucirene \\ Rangel-Lyne ${ }^{3}$ \\ ${ }^{1}$ Universidad del Noreste (UNE), a173529@une.edu.mx, Av. Miguel Hidalgo 6315, Nuevo Aeropuerto, \\ 89337 Tampico, Tamps. 8332303830 \\ 2 Universidad del Noreste (UNE), a172833@une.edu.mx, Av. Miguel Hidalgo 6315, Nuevo Aeropuerto, \\ 89337 Tampico, Tamps. 8332303830
}

${ }^{3}$ Universidad Autónoma de Tamaulipas (UAT)-Universidad del Noreste (UNE), rangel.lyne@gmail.com, Adolfo López M. S/N, Universidad, 89109 Tampico, Tamps. - Av. Miguel Hidalgo 6315, Nuevo Aeropuerto, 89337 Tampico, Tamps. 8332303830

\footnotetext{
Información del artículo revisado por pares

Fecha de aceptación: junio-2021

Fecha de publicación en línea: diciembre-2021

DOI: https://doi.org/10.29105/vtga7.2-11
}

\section{Resumen}

El profesionista actual se encuentra construyendo el paradigma 4.0 en el que la tecnología no solamente se limita al uso de la Internet y la conectividad virtual sino que abre un panorama de oportunidades más amplio a través de la Inteligencia Artificial. Uno de los sectores que más demandan estar actualizado es el del comercio internacional. Los profesionistas invierten parte de su tiempo en consultar actualizaciones pero los tiempos dedicados a estas tareas necesarias se limitan a factores de tiempo, esfuerzo, utilidad, facilidad de uso y economía en consecuencia surgen problemas de estrés laboral y al mismo tiempo repercute negativamente en la productividad y competitividad profesional. El evolucionar en la búsqueda y obtención de información con voz artificial y brindar un filtro de información confiable de acuerdo con los requerimientos del profesionista 4.0 sugiere un reto conveniente. Se realizó un estudio exploratorio descriptivo con base en una encuesta autoadministrada de manera electrónica a 495 profesionistas del sector de comercio internacional y aduanas. La mayor parte confirma una necesidad de optimizar sus tiempos invertidos en actualizaciones, además de evidenciar una aceptación por una búsqueda más eficiente de información ad hoc a sus necesidades. Además, se alerta de una probable inversión económica a cambio de ello. Una aplicación móvil con asistente de voz inteligente para capacitación del 
profesionista del paradigma 4.0 permitiría mejorar el rendimiento de todos los stakeholders del comercio internacional con posibilidades de aplicarse en otros sectores de semejante exigencia.

Palabras clave: paradigma 4.0, voz inteligente, capacitación.

\section{Abstract}

The current professional is building the new 4.0 paradigm in which technology is not limited to the use of the internet and virtual connectivity; rather, it opens broader opportunities through artificial intelligence. One of the sectors with the highest updating and training demands is related to the international commerce. Such professionals spend part of their time checking for updates; but the time devoted to these necessary tasks is limited to factors of time, effort, utility, ease of use and economy. Consequently, stress problems arise; negatively impacting on productivity and professional competitiveness. Therefore, evolving in the search for and obtaining information with artificial voice, as well as providing a reliable information filter in accordance with the requirements of the 4.0 professional, is suggested as a suitable challenge. A descriptive exploratory study was carried out based on a self-administered electronic survey of 495 professionals from the international commerce and customs sector. Most of them confirm a need to optimize their time invested in updates, in addition to showing an acceptance for a more efficient search for information ad hoc to their needs. In addition, they exposed a purchase intention to get this kind of services. Finally, a mobile application with an intelligent voice assistant for training the paradigm 4.0 professional, would allow to improve the performance of all the stakeholders of international commerce, with the possibility of being applied in other sectors of similar demand.
Keywords: 4.0 paradigm, artificial intelligence voice, training.

JEL: 035, Q55.

\section{INTRODUCCIÓN}

El paradigma 4.0 o "la Industria 4.0" tiene como característica la alta automatización de procesos y la digitalización del sector industrial (Coskun, 2019). El profesionista actual se encuentra construyendo dicha revolución en la que la tecnología no solamente se limita al uso de la Internet y la conectividad virtual sino que abre un panorama de oportunidades más amplio a través de la Inteligencia Artificial (Bacre, 2020). "El término inteligencia artificial (IA) se refiere a la capacidad de emular las funciones inteligentes del cerebro humano" (Badaró, Ibañez y Agüero, 2013).

La Inteligencia Artificial ha sido aplicada para el desarrollo de asistentes por voz, esto permite al usuario dictar comandos de manera verbal pues el reconocimiento de voz alerta al sistema para procesar la información y responder al requerimiento. Estos avances han permitido coadyuvar el día a día del profesional actual (Kim, 2020).

El mercado laboral cuenta con mayores exigencias, sobre todo cuando la labor requiere de una constante y especializada capacitación. Algunas innovaciones han desarrollado contactos virtuales ad hoc a las necesidades laborales (Mundo Aduanero, 2021). Ante los retos de estar capacitado para mantenerse al tanto de las nuevas actualizaciones en el ramo competente y el combinar la vida personal y familiar surge la necesidad de proponer nuevas formas de capacitación óptimas a las necesidades de cada área.

Uno de los sectores que más demandan estar actualizado es el comercio internacional. Los profesionistas de este sector requieren capacitarse constantemente con el ánimo de prevenir infortunios y estar atentos a cualquier cambio o reforma. Esta capacitación - actualización de información se torna compleja al enfrentar un vínculo de índole 
internacional. El presentar los documentos, adecuaciones o trámites para operaciones internacionales demanda una constante $y$ precisa capacitación (Madrid y Moreno, 2019). Las personas involucradas en este sector invierten comúnmente parte de su tiempo en consultar actualizaciones y los tiempos dedicados a estas tareas necesarias se limitan a los recursos de: tiempo, esfuerzo y economía.

A partir de ello surgen problemas como el estrés laboral (Astorquiza-Bustos; Castillo y Gómez-Mejía, 2020). "El estrés laboral se ha considerado una experiencia subjetiva de la persona, producida por la percepción de que existen demandas excesivas o amenazantes difíciles de controlar y que pueden tener consecuencias negativas para ella" (Peiro y Rodríguez, 2008), lo que al mismo tiempo repercute negativamente en la productividad y en la competitividad profesional, así lo argumentan estudios previos coincidentes: "el estrés laboral afecta directamente a la productividad del trabajador" (Halkos y Bousinakis, 2010). El evolucionar a la búsqueda y obtención de información con voz artificial y brindar un filtro de información confiable de acuerdo con los requerimientos del profesional exige un reto puntual.

La facilidad en el método de búsqueda de información inteligente a través de un asistente de voz es útil a partir de la optimización en tiempos y reducción de estrés laboral que éste puede llegar a representar.

En general esta innovación podría llegar a ser un apoyo para lograr desarrollar una vida con mayor calidad pues se enfoca en un recurso no renovable ligado al ser humano que tiene un alto impacto en todas las áreas de la vida cotidiana: el factor tiempo. Si se optimiza este factor y nos avocamos en el capital humano se esperaría un efecto positivo en la cadena de suministro porque al mantener actualizado al capital humano sin contratiempos se mantiene el nivel de especialización que exigen las cadenas de suministro en la actualidad e incluyendo desde los niveles operativos hasta los niveles gerenciales o estratégicos.

Además, al disminuir el esfuerzo invertido en este tipo de búsquedas se elevaría las posibilidades de mantenerse productivo y competitivo por medio de un fácil acceso a la capacitación con voz inteligente.

Desde otra perspectiva, iniciar con el desarrollo de propuestas innovadoras para el profesionista del paradigma 4.0 incluye una orientación inclusiva en el que se les brinda a las personas con capacidades diferentes y la oportunidad de obtener información oficial por cuenta propia de una manera más fácil.

Antes de proponer una iniciativa tecnológica este estudio se interesa en cómo es la postura de este segmento de profesionistas del paradigma 4.0 ante la propuesta de optimizar los factores de tiempo, esfuerzo y utilidad con una facilidad de uso para las actualizaciones que requieren.

Se realiza un estudio exploratorio con el objetivo de conocer la aceptación potencial que tendría este tipo de propuesta innovadora antes de exponerla en el mercado.

Con base en la observación y estudios empíricos previos el segmento estudiado obedece al profesional del comercio exterior al ser uno de los segmentos que se evidencia como uno de los que mantiene mayores demandas de actualizaciones constantes con tiempos restringidos de capacitación.

El desarrollo del estudio se presenta de la siguiente manera: primero se presentan breves antecedentes del contexto estudiado para después desarrollar la metodología, los resultados y conclusiones del análisis.

2. MARCO TEÓRICO

2.1. Migrando de texto a voz

La síntesis de voz es la generación de voz por medios artificiales. La producción de sonido para simular el habla humana se conoce como síntesis de bajo nivel. La síntesis de alto nivel se ocupa de la conversión de texto o símbolos escritos en una representación abstracta de la señal acústica deseada y adecuada para impulsar un sistema de síntesis de bajo nivel 
(Encyclopaedia Britannica, 2021). Con esta información se concreta que la síntesis de voz consiste en dos puntos clave: la síntesis de alto nivel y una síntesis de bajo nivel que Estos sistemas de síntesis de voz tienen diferentes cualidades como son la naturalidad y la inteligibilidad. La naturalidad hace que el audio de salida esté muy cerca de la voz humana mientras que la inteligibilidad es el grado de entendimiento que tiene el audio. El sintetizador de voz ideal debe ser natural e inteligible (Gironda, 2020). La síntesis de voz debe ser poseedora de naturalidad y de la intangibilidad con el objetivo de que se asemeje a la voz humana y sea del agrado de los consumidores.

"Text-to-Speech" comúnmente conocida como TTS es la tecnología que permite reproducir el habla humana de forma artificial. Esta tecnología es capaz de recibir un texto y reproducirlo con una voz artificial sintetizada (Innovando, 2021). El uso de esta herramienta tecnológica permitirá que los usuarios disfruten el escuchar un texto de su interés cuando éstos realizan actividades.

La tecnología de TTS es la capacidad de un sistema para reproducir un texto impreso en lenguaje hablado, es decir, son sistemas que realizan la conversión de textos escritos a una voz sintética (Giusti, Lira, Rodríguez-Vuan y Villarreal, 2016).

Existen varias aplicaciones que convierten el texto a voz y funcionan mediante software o hardware. Dependiendo de la complejidad del texto las aplicaciones pueden leerlo de manera natural o de manera muy robotizada porque intervienen diversas reglas de combinaciones de letras, entonaciones y duraciones de las palabras. Con respecto a la información que se brinda existen aplicaciones móviles que ayudan a las personas con problemas visuales, de comprensión lectora e incluso de tiempo para leer por ellas libros electrónicos y cualquier otro documento digital, página web o correo funcional y práctica para los usuarios que buscan optimizar su tiempo (Revista Gadget, 2020). logran una síntesis de voz adecuada para el usuario.

electrónico. Hay aplicaciones que utilizan un sistema Text-to-Speech (TTS) capaz de convertir un texto normal en una voz artificial (Marquina, 2020).

\subsection{Migrando a hacia la búsqueda por voz} La búsqueda por voz funciona integrando dos aspectos importantes: la búsqueda predictiva y el altavoz inteligente: "La búsqueda predictiva consiste en la previsión semántica de necesidades a través de motores de búsqueda que arrojan resultados con base en el contexto actual, el comportamiento histórico, patrones de conducta y solicitudes activas de información." (Jiménez Cubría, 2015). Es importante conjugar el altavoz inteligente que es el asistente virtual que funciona por medio de la búsqueda por voz y la búsqueda predictiva se refiere aquella función que arroja los términos más solicitados por los usuarios.

Publicaciones en revistas especializadas discuten acerca de las ventajas de la Inteligencia Artificial aplicada a la búsqueda por medio de la voz. El altavoz inteligente es capaz de escuchar al usuario para atender sus peticiones que pueden ser de cualquier tipo.

El asistente virtual tiene un corazón tecnológico que es un software capaz de entender las diferentes maneras de expresarse y de pronunciar las palabras de manera natural. Para ello se parte de una base cognoscitiva que se agrega a los algoritmos más complejos basados en la tecnología de formación continua relacionada con gustos, hábitos y aficiones, así como de un ecosistema doméstico-familiar y apreciada como una herramienta

Esta nueva tecnología consiste en el reconocimiento del lenguaje hablado que permite a los usuarios realizar búsquedas sin 
tener que escribir los términos en las cajas de los motores de búsqueda. Estas búsquedas se realizan a través de los denominados asistentes de voz programados con Inteligencia Artificial (IA), Machine Learning y tecnología de reconocimiento con el fin de ejecutar una serie de funciones y/o servicios solicitados por un usuario (Selligent, 2020).

Estos dos elementos se complementan ya que para que el altavoz inteligente funcione correctamente $\mathrm{y}$ arroje los resultados esperados debe tener elementos base de acuerdo a lo que es del interés del usuario.

\subsection{Alexa}

Alexa es un ejemplo avanzado de asistente virtual controlado por voz creado por Amazon y lanzado en noviembre de 2014 en conjunto con una línea de altavoces inteligentes "Echo". Tal nombre (Alexa) fue elegido por tener una consonante fuerte al principio e incluir una " $x$ ", algo que haría que el asistente reconociese más fácil su nombre y también en honor a la Biblioteca de Alejandría (Fernández, 2021).

Según Fernández (2021) el funcionamiento de Alexa es muy parecido al de otros asistentes como Google Assistant, Siri y Cortana. Se comienza llamándole por su nombre para que después el altavoz o dispositivo en el que esté integrado lo escuche, entonces se envía un comando con la voz del usuario y el asistente reconocerá la pregunta respondiendo a la misma.

La voz de Alexa no proviene de la voz de una persona real. La voz de Alexa fue generada desde las reglas de Text-to-Speech o TTS (texto a voz) más Inteligencia Artificial (IA). Aunque algunos asistentes de voz en el pasado han sonado más mecánicos, Amazon está realizando esfuerzos importantes para que dicho asistente se escuche cada vez más parecido a una persona (EI Locutorio, 2021).

Alexa es un asistente virtual que Amazon lanzó al mercado y funciona por búsqueda de voz. A pesar de que la voz no es de una persona real es una voz similar a la del humano y la distingue de otras aplicaciones que generalmente utilizan voces robotizadas, lo que para muchos usuarios no es ameno para sus oídos o gustos.

2.4. Modelo de Aceptación Tecnológica (TAM)

El Modelo de Aceptación Tecnológica (TAM) es eficiente y ha sido empleado con éxito en diversas investigaciones empíricas. Davis (1989) fue el pionero en el desarrollo de este modelo con base en la Teoría de Acción de la Razón (TRA).

"EI TAM fue especialmente diseñado para predecir la aceptación de los sistemas de información por los usuarios en las organizaciones" (Yong Varela, Rivas Tovar y Chaparro, 2008).

Según Davis (1989) el propósito principal del TAM es explicar los factores que determinan el uso de las TIC's por un número importante de usuarios. EI TAM sugiere que la utilidad y la facilidad de uso son determinantes en la intención que tenga un individuo para usar un sistema.

Dicho modelo ha sido el más aceptado por los estudiosos de las Tecnologías de la Información (TIC) debido a su efectivo poder predictivo (Yong Varela, Rivas Tovar y Chaparro, 2008).

Algunas de las variables principales que se incluyen en el TAM son: la utilidad percibida y facilidad de uso percibida. La utilidad percibida se refiere al grado en la que una persona tendrá una mejora dentro de su trabajo. La facilidad de uso percibida se relaciona con el grado en que una persona considera que está utilizando un sistema y realizará menos trabajo para la consecución de sus tareas (Davis, 1989).

"El principal propósito del TAM es indagar las consecuencias de los factores externos en cuanto a la utilidad (Perceived Usefulness) y la facilidad de uso percibidas (Perceived ease-of-use), para adelantar o predecir el uso de las TIC" (Yong Varela, Rivas Tovar y Chaparro, 2008).

3. MÉTODO

La presente investigación tuvo un alcance exploratorio descriptivo de corte transversal. 
Obedeciendo al objetivo de explorar la aceptación de esta clase de tecnologías con Inteligencia Artificial para la capacitación de los profesionistas del paradigma 4.0 se realizó una encuesta electrónica vinculada a las variables de tiempo, utilidad, esfuerzo, facilidad de uso y factor económico.

Se realizó una prueba piloto con 25 personas relacionadas con el área para poder cuestionar acerca de la comprensión de las preguntas directas.

Después de hacer los ajustes pertinentes se procedió a lanzar la encuesta electrónica a la muestra final.

Las preguntas contenidas en la encuesta tuvieron una base dicotómica. La pregunta con relación al factor tiempo fue la siguiente: ¿Le gustaría optimizar su tiempo al mismo tiempo que se actualiza en materia de comercio internacional? Esta pregunta incluyó la palabra optimizar simultánea al planteamiento de una actualización en materia de comercio exterior con lo que en un primer momento se expone la asociación con el encuestado.

La segunda pregunta corresponde a la utilidad y esfuerzo: ¿Le agradaría obtener actualizaciones diarias relacionadas con el comercio internacional de manera eficiente, sólo mostrando los aspectos relevantes, omitiendo información general? En esta pregunta se invita al encuestado a responder si sería agradable recibir diariamente actualizaciones pertinentes con su labor. En otras palabras: recibir actualizaciones que su labor requiere sin la necesidad de tener que realizar esfuerzos por entrar, buscar y filtrar la información.

La tercera pregunta se vincula con el factor facilidad de uso: ¿Le gustaría una app de comercio internacional en donde se tengan filtros de búsqueda para la información y así agilizar la búsqueda? Este planteamiento expone una facilidad de uso sin mencionar el nombre de la variable, únicamente describiendo lo que representaría de una manera general.
Con respecto a la cuarta pregunta con base en el factor económico: ¿Pagarías una membresía mensual? Se permitió cuestionar de manera muy directa al profesionista acerca de su disposición a invertir mensualmente a cambio de obtener esta clase de actualizaciones que optimizan el tiempo son útiles para su actualización profesional y que a la par se propondría como una aplicación (app) de fácil uso y acceso.

Finalmente se encuestó al segmento de profesionistas del comercio exterior, logística, derecho aduanero, derecho mercantil, negocios internacionales, maestros del área, trabajadores de navieras, terminales portuarias, agencias aduanales, aduanas, recintos fiscalizados, comercializadoras, exportadores e importadores.

La muestra se conformó por 495 profesionistas de Tampico, Altamira, Ciudad Madero y municipios pertenecientes al Sur del estado de Tamaulipas.

A continuación se presentan los resultados del estudio exploratorio.

4. RESULTADOS

De acuerdo con los objetivos del estudio se realizaron preguntas con respecto a los factores de tiempo, utilidad, esfuerzo y facilidad de uso.

Como descrito previamente se agregó a forma de complemento la pregunta al factor económico. Con esto en un momento inicial se consideraría el potencial de desarrollar una propuesta formal que responda a la situación actual de los profesionistas en el paradigma 4.0.

Los encuestados coinciden en su mayoría en los siguientes resultados:

En relación con la pregunta del factor tiempo la respuesta evidencia la necesidad de optimizar los tiempos, teniendo casi el $100 \%$ de aceptación (Figura 1). 
Figura 1. Pregunta de factor tiempo.

¿Le gustaría optimizar su tiempo al mismo tiempo que se actualiza en materia de comercio internacional?

$3.1 \%$

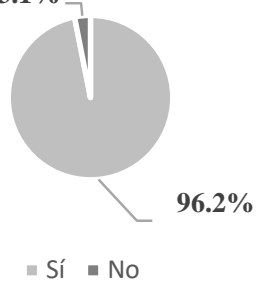

Fuente: Elaboración propia.

Con respecto a los factores de utilidad y esfuerzo se denota el requerimiento de obtener información ad hoc a las actualizaciones pertinentes y elegidas por el usuario de una manera eficiente (Figura 2).

Figura 2. Pregunta factor utilidad y esfuerzo.

¿Le agradaría obtener actualizaciones diarias relacionadas con el comercio internacional de manera eficiente, sólo mostrando los aspectos relevantes, omitiendo información general?

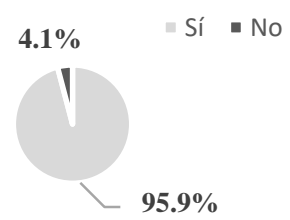

Fuente: Elaboración propia.

Se cuestionó acerca de la aceptación de la propuesta a través de una aplicación móvil especializada en comercio internacional que contenga los filtros de búsqueda para realizar la personalización del servicio informativo. Las respuestas fueron en su mayoría positivas y dando la pauta para proponer este desarrollo vía una aplicación móvil que pueda ser programada según las preferencias del profesionista.
Figura 3. Facilidad de uso.

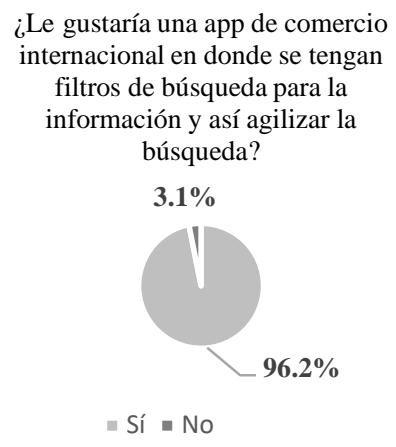

Fuente: Elaboración propia.

Además de contar con una aceptación se cuestionó acerca de la disposición para pagar una membresía por el uso de tal aplicación, obteniendo más del $50 \%$ de valoraciones positivas y el $36,7 \%$ de valoraciones posibles. Solamente el $6,4 \%$ respondió que no estaría dispuesto a pagar por recibir el servicio (Figura 4).

\section{Figura 4. Factor económico}

¿Pagarías una membresía

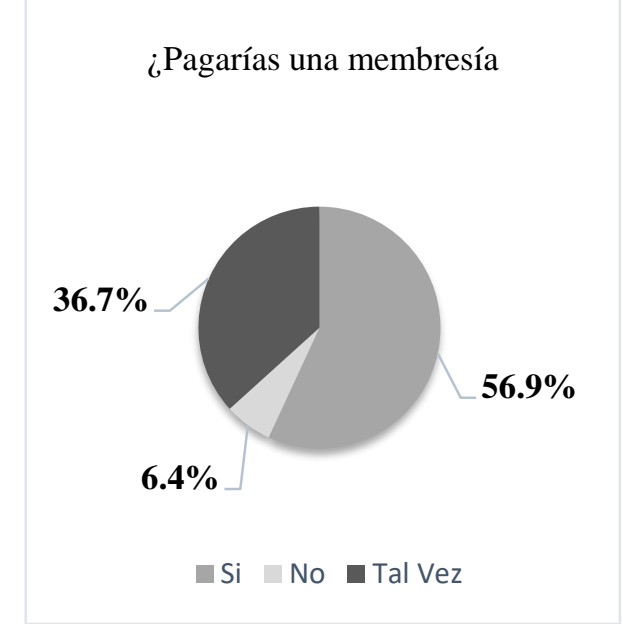

Fuente: Elaboración propia.

Con base en los resultados de las encuestas realizadas se puede observar que la mayoría de los encuestados relacionados con alguna ocupación referente al comercio internacional (más del 95\%) están interesados en una herramienta que les sea útil como medio informativo en el ámbito del comercio 
exterior y que además les ayude a optimizar el tiempo.

Complementando con la literatura revisada sería factible realizar la propuesta de una aplicación móvil con base en asistencia de voz que facilite y optimice más el tiempo invertido por el usuario ante la necesidad de estar actualizado.

Este estudio pionero nos lleva a entender que la aplicación puede ser aceptada por el mercado debido al interés que se mostró ya que cubre con las necesidades de capacitación e información de las personas que se desarrollan dentro de este ámbito.

\section{CONCLUSIONES}

Con relación en las variables confirmadas desde el modelo TAM (facilidad de uso y utilidad percibida) éstas pueden sugerirse en las preguntas planteadas en un nivel apenas exploratorio. A más del $95 \%$ de los encuestados les agradaría una opción para optimizar su tiempo y de la misma forma adquirir nuevos conocimientos sobre el comercio.

Ésta se propone como una probable solución a los tiempos poco efectivos que se provocan al momento de realizar la búsqueda, depuración y análisis de la información.

Con ello se busca aligerar el estrés, elevar la calidad de vida y optimizar el factor tiempo al reducir el esfuerzo y aumentar la utilidad de uso efectivo en materia de capacitaciones y actualizaciones especializadas. A su vez todo esto sugiere un efecto positivo en la productividad y competitividad del profesionista.

Con la facilidad de acceso a la información se busca también tener un mejor control de la capacitación del personal a lo largo de la cadena logística con lo que además se coadyuvaría a reducir el número de infracciones, multas o procedimientos administrativos con potencial de causar mayores mermas en los procesos.

Debido a que todos los niveles dentro de la cadena logística podrán hacer uso de la información se propone esta innovación en el mercado laboral ya que permitirá a los usuarios obtener información útil sin importar el lugar o la hora en la que se consulte, siendo inclusiva para personal con alguna capacidad diferente o que no desee permanecer largo tiempo extra ante la pantalla al buscar información.

Finalmente esta aplicación móvil con asistente de voz inteligente para capacitación del profesionista del paradigma 4.0 permitiría mejorar el rendimiento de todos los stakeholders de la cadena logística del comercio internacional con posibilidades de aplicarse en otros sectores de semejante exigencia. 


\section{REFERENCIAS}

Astorquiza-Bustos, B. A., Castillo, M. C. y Gómez-Mejía, A. (2020). Estrés laboral en el mercado laboral: una aplicación de metodologías de medición difusa para el caso colombiano. En: https://revistas.udea.edu.co/index.php/lecturasdeeconomia/article/view/339564

Bacre, G.D., Martínez M.A. y Leal, N.M. (2020). Propuesta de modelo para migrar hacia la educación 4.0. Revista Vinculatégica. En: http://www.web.facpya.uanl.mx/vinculategica/Vinculategica6_2/10_Bacre_Martinez_Leal.pdf Badaró, S., Ibañez, L. J. y Agüero, M. J. (2013). Sistemas Expertos: Fundamentos, Metodologías y Aplicaciones. Ciencia y Tecnología.

Davis, F. D. (1989). Perceived usefulness, perceived ease of use, and user acceptance of information technology. MIS quarterly, 319-340.

El Locutorio. (03 de MARZO de 2021). ¿De quién es la voz de Alexa, la asistente virtual de Amazon? https://www.ellocutorio.com/quien-es-la-voz-del-asistente-virtual-alexa-de-amazon/

Fernández, Y. (2021). XAKATA BASICS. Qué es Alexa, qué puedes hacer con él y qué dispositivos son compatibles. En: https://www.xataka.com/basics/que-alexa-que-puedes-hacer-que-dispositivoscompatibles

Gironda, R. (2020). Tecnologías de reconocimiento de voz avanzado. Diferentes alternativas Text to Speech. En: https://blog.enzymeadvisinggroup.com/tecnolog\%C3\%ADas-reconocimiento-vozavanzado-text-to-speech

Giusti, R., Lira, M., Rodríguez-Vuan, A. y Villarreal, G. (2016). Accesibilidad de los contenidos en un repositorio institucional: análisis, herramientas y usos del formato EPUB. e- Ciencias de la Información, 3. En: https://www.redalyc.org/articulo.oa?id=476852098004

Guzmán, B., Del Carmen, D., Martínez Mercado, De los Ángeles, M., Leal Rendón y Nury, M. (2020). Propuesta de modelo para migrar hacia la educación 4.0. Latindex, 1. En: http://www.web.facpya.uanl.mx/vinculategica/Vinculategica6_2/10_Bacre_Martinez_Leal.pdf Halkos, G. y Bousinakis, D. (2010). The effect of stress and satisfaction on productivity. International Journal of Productivity and Performance Management, 59(5), 415-431.

INNOVANDO. (2021). Síntesis del habla. https://innovan.do/2015/04/18/que-es-text-to-speechsintesis-del-habla-definicion/

ITM Platform (23 de marzo de 2021). Fases de un proyecto de desarrollo de aplicación móvil. https://www.itmplatform.com/es/blog/fases-de-un-proyecto-de-desarrollo-de-aplicacion-movil/ Jiménez Cubría, A. G. (2015). Merca2.0. Obtenido de La búsqueda predictiva: ¿Una nueva herramienta de marketing? En: https://www.merca20.com/la-busqueda-predictiva-una-nuevaherramienta-de-marketing/

Kim, T. (2020). Short research on voice control system based on artificial intelligence assistant. In: International Conference on Electronics, Information, and Communication (ICEIC), pp. 1-2

Marquina, J. (2020). 11 aplicaciones móviles que leen en voz alta tus libros y casi todo tipo de documentos. En: https://www.julianmarquina.es/aplicaciones-moviles-que-leen-en-voz-alta-tuslibros-y-casi-todo-tipo-de-documentos/

MERCA2.0, (2015). ¿Una nueva herramienta de marketing?. https://www.merca20.com/labusqueda-predictiva-una-nueva-herramienta-de-marketing/

Madrid, S. D. P. C. y Moreno, M. P. (2019). Metaanálisis de los artículos sobre estrés laboral docente en el periodo 2013-2017. RECIMUNDO: Revista Científica de la Investigación y el Conocimiento, 3(1), 522-554.

Mundo Aduanero (2021). Recuperado de: https://www.mundoaduanero.mx 
Peiro, J. M. y Rodríguez, I. (2008). Estrés laboral, liderazgo y salud organizacional. Papeles del Psicólogo, vol. 29, núm. 1, 69.

Revista Gadget. (2020). Revista Gadget. Obtenido de Altavoces inteligentes: Qué son y para qué sirven. http://www.revista-gadget.es/reportaje/altavoces-inteligentes-que-son/

SEGOB. (2019). BREVE HISTORIA DEL PERIÓDICO OFICIAL EN MÉXICO. Obtenido de Diario Oficial de la

Federación:

https://www.dof.gob.mx/historia.php\#: :text=El\%20Diario\%200ficial\%20de\%20la,que\%20\%C3\%A 9stos\%20sean\%20observados\%20y

Selligent. (2020). VENTAJAS E INCONVENIENTES DEL VOICE SEARCH, LOS BUSCADORES DEL FUTURO. Obtenido de Selligent: https://www.selligent.com/es/blogs/consejos/ventajas-e-inconvenientesdel-voice-search-los-buscadores-del-

futuro\#: :text=Esta\%20nueva\%20tecnolog\%C3\%ADa\%20consiste\%20en,de\%20los\%20motores\%2 Ode\%20b\%C3\%BAsqueda.

Encyclopaedia Britannica. (2021). The Editors of the Encyclopaedia Britanica. https://www.britannica.com/science/vocalization

Uribe Vélez, J., Ávila Roa, L. y Chacón Ramírez, E. A. (2020). Sistema de gestión de energía bajo el paradigma de Industria 4.0. Revista Ingenio, 1.

Yong Varela, L. A., Rivas Tovar, L. A. y Chaparro, J. (Abril de 2008). Revistas UNAL. Obtenido de Modelo de aceptación tecnológica (TAM): un estudio de la influencia de la cultura nacional y del perfil del usuario en el uso de las TIC: https://revistas.unal.edu.co/index.php/innovar/article/download/29202/34795 